\title{
Partial entrainment in the finite Kuramoto-Sakaguchi model
}

\author{
Filip De Smet, Dirk Aeyels \\ SYSTeMS Research Group, Dept. of Electrical Energy, Systems and Automation, \\ Ghent University, Technologiepark-Zwijnaarde 914, 9052 Zwijnaarde, Belgium
}

\begin{abstract}
Although modifications of the Kuramoto model have been the subject of extensive research, the model itself is not yet fully understood. We offer several results and observations, some analytic, others through simulations. We derive a sufficient condition for the existence of a solution exhibiting partial entrainment with respect to a given subset of oscillators; the result also implies persistence of the entrainment behavior under perturbations.

The critical values of the coupling strength, defining the transitions between different forms of partial entrainment, are predicted by an analytical approximation, based on the fact that oscillators with large differences in their natural frequencies have little influence on each other's entrainment behavior; the predictions agree with the actual values, obtained by simulations.

We indicate (by simulations) that entrainment can disappear with increasing coupling strength, and that, in arrays of Josephson junctions, a similar phenomenon can be observed, where it is also possible that a junction leaving one entrained subset joins another entrained subset.
\end{abstract}

Pacs numbers: 05.45.Xt, 89.75.Fb, 74.81.Fa

Key words: coupled oscillators; Kuramoto model; entrainment

\section{Introduction}

The Kuramoto model [6] is a prototype model for the study of systems of coupled oscillators. A discussion of illustrative examples, such as flashing fireflies, coupled laser arrays and pacemaker cells in the heart, can be found in [17]. We will consider the extension introduced in [15] by Kuramoto and Sakaguchi, 
which is described by the differential equations

$$
\dot{\theta}_{i}(t)=\omega_{i}+\frac{K}{N} \sum_{j=1}^{N} \sin \left(\theta_{j}(t)-\theta_{i}(t)-\alpha\right),
$$

$\forall i \in\{1, \ldots, N\}, \forall t \in \mathbb{R}$, where $N$ is the number of oscillators, $K \geq 0$ is the coupling strength, $|\alpha| \leq \frac{\pi}{2}$ and the $\omega_{i}$ are drawn from a distribution $g$. (The Kuramoto model corresponds to the case $\alpha=0$.) The parameters $\omega_{i}$ represent the natural frequencies of the oscillators and determine the behavior of the system for $K=0$. For a system with an infinite number of oscillators $\mathrm{Ku}$ ramoto and Sakaguchi showed that there is a critical value $K_{c}$ of the coupling strength above which a solution exists exhibiting partial synchronization. For $K>K_{c}$ this solution is characterized by two different groups of oscillators; those in the first group are locked at a frequency $\Omega$ while the other oscillators are moving with long term average frequencies different from $\Omega$. The stability properties of this solution are not yet fully understood.

The analysis by Kuramoto and Sakaguchi cannot simply be transposed to the case with a finite number of oscillators. Analytical results are hard to obtain and treat special cases such as identical natural frequencies [18] or the case of complete phase locking behavior and its stability properties [2, 5]. Most research focuses on simulations $[7,12]$ and is concerned with modifications such as an alternative interaction structure $[4,11,3,13,10,9,14]$, although the unmodified Kuramoto model has also received some attention in recent years $[7,12,8]$. For an overview see e.g. $[16,1]$.

In this paper we will investigate general partial entrainment in the KuramotoSakaguchi model with non-identical natural frequencies, providing both analytical results and simulations. In the next section we describe the general behavior of the Kuramoto-Sakaguchi model as it is observed in simulations with small $N$, and in support of these observations we formulate a sufficient condition (for general finite $N$ ) for the entrainment of a given subset of the population of oscillators. The proof implies persistence of the entrainment behavior under perturbations in the initial condition and the result remains non-trivial in the limit $N \rightarrow \infty$.

Section 4 deals with an estimation for the critical values of the coupling strength defining the transitions between different forms of partial entrainment for the case $\alpha=0$. In section 5 we illustrate the phenomenon for which, for both the Kuramoto-Sakaguchi model (with general $\alpha$ ) and a system of Josephson junction arrays, entrainment may disappear with increasing coupling strength. 


\section{The general scenario}

Let $\theta$ be a solution of the system (1) and let $S_{\mathrm{e}} \subset\{1, \ldots, N\}$ be non-empty. Definition 1. If

$$
\exists C>0:\left|\theta_{i}(t)-\theta_{j}(t)\right|<C, \quad \forall t \geq 0, \forall i, j \in S_{\mathrm{e}}
$$

then the solution $\theta$ exhibits partial entrainment with respect to $S_{\mathrm{e}}$, and $S_{\mathrm{e}}$ is called an entrained subset.

Notice that according to this definition (which slightly differs from the one in [2], where the system was said to exhibit partial entrainment if at least two oscillators exist with bounded phase differences) there is always a trivial form of entrainment corresponding to the singletons $\{i\} \subset\{1, \ldots, N\}$. Partial entrainment with respect to the entire population is called full entrainment.

Simulations indicate that, for most values of the natural frequencies and the coupling strength, the entrainment behavior is independent of the initial condition - allowing us to refer to the entrainment behavior of the system - and furthermore that for each oscillator the long term average frequency converges to a constant (i.e. $\omega_{i}^{\lim } \triangleq \lim _{t \rightarrow \infty} \frac{\theta_{i}(t)}{t}$ exists), also independent of the initial condition for most parameter values and — as follows from definition 1 equal for all oscillators in the same entrained subset.

\subsection{Stepwise entrainment buildup for increasing $K$}

For most simulations of $(1)$ with $N$ small and $|\alpha|$ small $(\lesssim 0.5)$ the entrainment behavior in terms of the coupling strength can be described as follows. If all $\omega_{i}$ are different then for $K=0$ the only entrained subsets are trivially the singletons $\{i\}, 1 \leq i \leq N$.

With increasing $K$, oscillators start to become entrained, enlarging sets already entrained. In general there are $N-1$ bifurcation values $K_{c, k}(k \in$ $\{1, \ldots, N-1\}$, each value representing a coupling strength where two entrained subsets (which subsets depends on the actual values of the $\omega_{i}$ ) merge. After $N-1$ transitions full entrainment occurs, which is investigated for $\alpha=0$ in $[2]$.

This scenario is clearly illustrated in figure 1, where the different long term average frequencies of the oscillators are plotted (horizontal axis) for varying coupling strength (vertical axis), for a system with $\alpha=0$, consisting of four oscillators with natural frequencies given by $\omega_{1}=-2.32, \omega_{2}=-0.89, \omega_{3}=$ 0.68 and $\omega_{4}=1.23$. Later on we indicate that this scenario might break down; 
see section 5 for details.

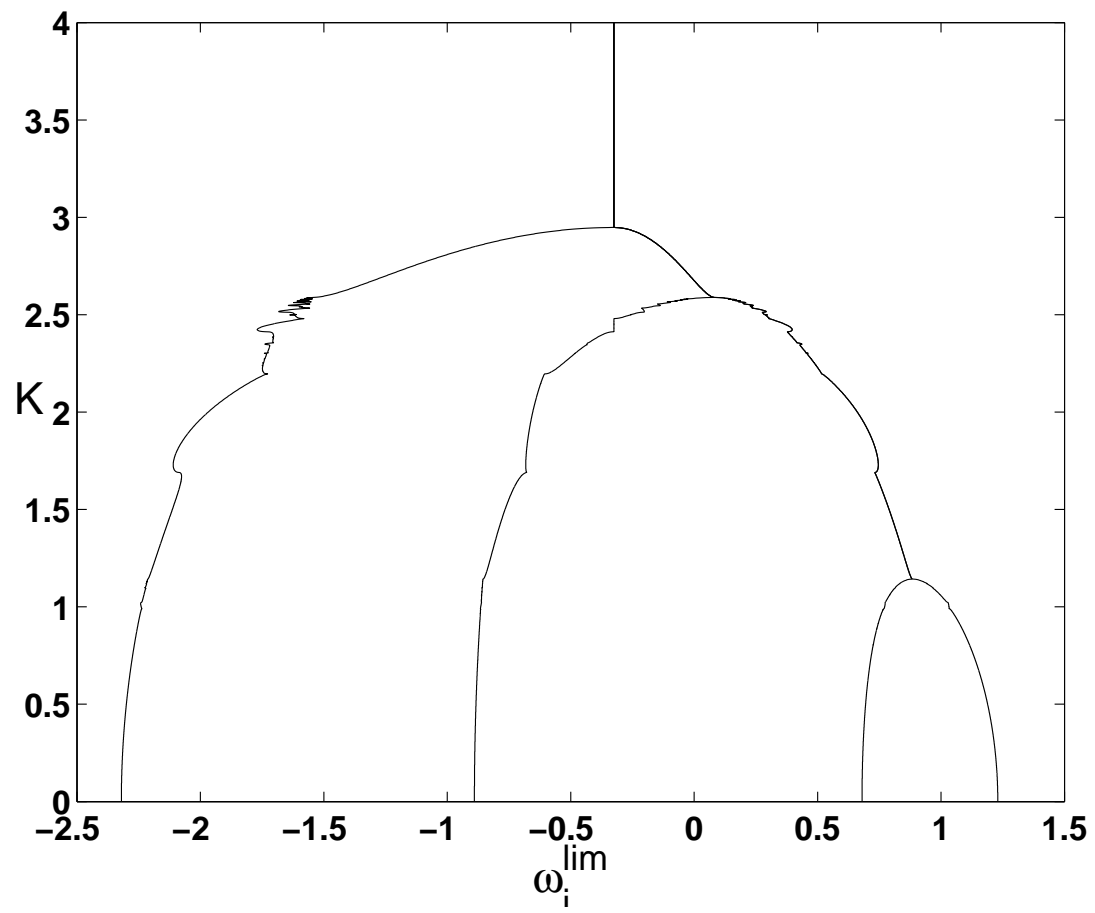

Fig. 1. Long term average frequencies for varying coupling strength for a system of four oscillators. Different forms of partial entrainment can be distinguished: from no entrainment for small $K$, to full entrainment for large $K$.

\subsection{A sufficient condition for partial entrainment}

In support of the scenario described in the previous section, which is based on simulations, we provide an analytical result (for general finite $N$ ), proving that the Kuramoto-Sakaguchi model is able to exhibit partial entrainment: we derive a sufficient condition for the existence of a solution exhibiting partial entrainment with respect to a given set of oscillators. This result is not to be considered as an attempt to estimate critical values for the coupling strength - this will be dealt with in section 4 - but as an analytical proof of the existence of partial entrainment.

We first formulate a preliminary result, pertaining to the model (1) with $\alpha=0$, to illustrate the technique of proof. A stronger but analytically more technical result follows.

Proposition 1. Let $S_{\mathrm{e}}$ be a proper subset of $\{1, \ldots, N\}$ with $M$ elements and such that $M>\frac{N}{2}$. Assume that

$$
\left|\omega_{i}-\omega_{j}\right|<K \sqrt{\frac{N}{M}}\left(\frac{4 M-2 N}{3 N}\right)^{\frac{3}{2}}, \quad \forall i, j \in S_{\mathrm{e}} .
$$


Then there exists a solution of (1) with $\alpha=0$ which exhibits partial entrainment with respect to $S_{\mathrm{e}}$.

Proof. For any $a \in\left(0, \frac{\pi}{2}\right)$ let $R_{a}$ denote the region

$$
R_{a}=\left\{\theta \in \mathbb{R}^{N}:\left|\theta_{i}-\theta_{j}\right| \leq a, \forall i, j \in S_{\mathrm{e}}\right\} .
$$

We will determine a value for $a$ for which $R_{a}$ is a trapping region for (1): we will show that the vector field points into $R_{a}$ at the boundary of $R_{a}$, such that any solution with an initial condition in $R_{a}$ remains in $R_{a}$.

Assume that for a particular $t_{0} \in \mathbb{R}$ the solution of (1) at time $t_{0}$ is located at the boundary of $R_{a}: \theta\left(t_{0}\right) \in R_{a}$ and $\theta_{i}\left(t_{0}\right)-\theta_{j}\left(t_{0}\right)=a$ for some $i, j \in S_{\mathrm{e}}$. From (1) it follows that

$$
\begin{aligned}
\dot{\theta}_{i}\left(t_{0}\right)-\dot{\theta}_{j}\left(t_{0}\right)=\omega_{i}-\omega_{j}-2 \frac{K}{N} \sin & \left(\frac{\theta_{i}\left(t_{0}\right)-\theta_{j}\left(t_{0}\right)}{2}\right) \\
& \times \sum_{k=1}^{N} \cos \left(\theta_{k}\left(t_{0}\right)-\frac{\theta_{i}\left(t_{0}\right)+\theta_{j}\left(t_{0}\right)}{2}\right) .
\end{aligned}
$$

In the summation we can bound the terms for which $k \in S_{\mathrm{e}}$ by

$$
\cos \left(\theta_{k}\left(t_{0}\right)-\frac{\theta_{i}\left(t_{0}\right)+\theta_{j}\left(t_{0}\right)}{2}\right) \geq \cos a
$$

since $\left|\theta_{k}\left(t_{0}\right)-\frac{\theta_{i}\left(t_{0}\right)+\theta_{j}\left(t_{0}\right)}{2}\right| \leq a$. (In fact $\left|\theta_{k}\left(t_{0}\right)-\frac{\theta_{i}\left(t_{0}\right)+\theta_{j}\left(t_{0}\right)}{2}\right| \leq \frac{a}{2}$, but this bound would result in more complicated calculations.)

If $k \notin S_{\text {e }}$ then $\cos \left(\theta_{k}\left(t_{0}\right)-\frac{\theta_{i}\left(t_{0}\right)+\theta_{j}\left(t_{0}\right)}{2}\right) \geq-1$, and thus

$$
\dot{\theta}_{i}\left(t_{0}\right)-\dot{\theta}_{j}\left(t_{0}\right) \leq \omega_{i}-\omega_{j}-2 \frac{K}{N} \sin \frac{a}{2}(M \cos a-(N-M)) .
$$

For $R_{a}$ to be a trapping region we need the right hand side to be negative. Minimizing this expression by choosing $a$ appropriately leads to $\sin \frac{a}{2}=\sqrt{\frac{2 M-N}{6 M}}$, resulting in

$$
\dot{\theta}_{i}\left(t_{0}\right)-\dot{\theta}_{j}\left(t_{0}\right) \leq \omega_{i}-\omega_{j}-K \sqrt{\frac{N}{M}}\left(\frac{4 M-2 N}{3 N}\right)^{\frac{3}{2}}<0,
$$

and thus for this value of $a R_{a}$ is a trapping region. Since $R_{a}$ is non-empty we can choose an initial condition in $R_{a}$ and the resulting solution of (1) will exhibit partial entrainment with respect to $S_{\mathrm{e}}$.

To extend proposition 1 we will invoke extra knowledge about oscillators not in $S_{\mathrm{e}}$ to provide a better bound for the term $\cos \left(\theta_{k}\left(t_{0}\right)-\frac{\theta_{i}\left(t_{0}\right)+\theta_{j}\left(t_{0}\right)}{2}\right)$ in (2) 
with $k \notin S_{\mathrm{e}}$. Although this term can attain its minimal value of -1 , if $\omega_{k}$ differs at least $2 K$ from $\frac{\omega_{i}+\omega_{j}}{2}$, then it cannot remain -1 and it will also attain positive values. Using this property, we will provide a condition for which a solution of (1), starting within a region $R_{a^{\prime}}$, with $a^{\prime} \in(0, a)$, cannot leave $R_{a}$. For the proof we refer to the appendix.

Proposition 2. Let $\left\{S_{1}, S_{2}, S_{3}\right\}$ be a partition of $\{1, \ldots, N\}$, with $S_{2}$ and $S_{3}$ possibly empty. Pick $m, M \in S_{1}$ such that $\omega_{m}=\min _{i \in S_{1}} \omega_{i}$ and $\omega_{M}=$ $\max _{i \in S_{1}} \omega_{i}$ and assume that $\left|\omega_{i}-\frac{\omega_{m}+\omega_{M}}{2}\right|>2 K>0, \forall i \in S_{2}$. Define $\tilde{\Theta}_{j}, \gamma_{1}$, $\tilde{\gamma}_{2}, \gamma_{3}$ and $T$ by

$$
\begin{gathered}
\tilde{\Theta}_{j} \triangleq \arccos \left(\frac{4 K}{4 K+\pi\left(\left|\omega_{j}-\frac{\omega_{m}+\omega_{M}}{2}\right|-2 K\right)}\right), \forall j \in S_{2}, \\
\gamma_{i} \triangleq \frac{\left|S_{i}\right|}{N}, \quad \forall i \in\{1,3\} \\
\tilde{\gamma}_{2} \triangleq \frac{1}{N} \sum_{j \in S_{2}} \cos \tilde{\Theta}_{j}, \\
T \triangleq \exp \left(\frac{2 K}{N} \sum_{j \in S_{2}} \frac{\sin \tilde{\Theta}_{j}-\tilde{\Theta}_{j} \cos \tilde{\Theta}_{j}}{\left|\omega_{j}-\frac{\omega_{m}+\omega_{M}}{2}\right|-2 K}\right),
\end{gathered}
$$

assume that $|\alpha|<\frac{\pi}{6}, \delta \triangleq \gamma_{1}^{2} \cos ^{2} \alpha-\left(\tilde{\gamma}_{2}+\gamma_{3}\right)^{2}>0$ and that the inequality

$\frac{\omega_{M}-\omega_{m}}{K} \leq \frac{4 \sqrt{3} T\left(\delta^{\frac{3}{2}}-\sqrt{3} \gamma_{1}|\sin \alpha| \delta\right)}{\left(3 T^{2}\left(\gamma_{1} \cos \alpha+\tilde{\gamma}_{2}+\gamma_{3}\right)+\gamma_{1} \cos \alpha-\tilde{\gamma}_{2}-\gamma_{3}\right)\left(2 \gamma_{1} \cos \alpha+\tilde{\gamma}_{2}+\gamma_{3}\right)}$

holds. Then there exists a solution of (1) exhibiting partial entrainment with respect to $S_{1}$.

\subsection{An asymptotic stability result}

We will show that, in case the entrained subset $S_{1}$ contains oscillators with equal natural frequencies, the submanifolds where the oscillators in $S_{1}$ have equal phases are asymptotically stable.

Assume all oscillators in $S_{1}$ have the same natural frequency: $\omega_{i}=\tilde{\omega}, \forall i \in S_{1}$, for some $\tilde{\omega} \in \mathbb{R}$. Let $S_{3}$ contain the oscillators with natural frequency equal to $\tilde{\omega}$ which are not included in $S_{1}$; then $S_{2}$ contains all oscillators with natural frequency different from $\tilde{\omega}$. (Notice that every solution of (1) will exhibit partial entrainment with respect to $S_{1}$ : if the difference $\theta_{i}(t)-\theta_{j}(t)$, with $i, j \in S_{1}$, would cross a multiple of $2 \pi$ at some time $t$, then $\dot{\theta}_{i}(t)-\dot{\theta}_{j}(t)=0$, and the oscillators $i$ and $j$ would coincide (modulo $2 \pi$ ) for all $t \in \mathbb{R}$.) Assume that $K$ is sufficiently small for the condition of proposition 2 on the oscillators 
in $S_{2}$ to be valid. As is shown in the appendix we can adapt the proof of proposition 2 to obtain the following.

Proposition 3. Assume that $\omega_{i_{1}}=\cdots=\omega_{i_{P}}=\tilde{\omega}$ for some $i_{1}, \ldots, i_{P} \in$ $\{1, \ldots, N\}$, with $2 \leq P \leq N$, and that no other oscillators have an $\omega_{i}$-value equal to $\tilde{\omega}$. Then for any $M \in \mathbb{Z}$, with $M>\frac{P}{1+\cos \alpha}$, there exists an $\epsilon>0$, such that $\forall K \in(0, \epsilon)$ the submanifolds defined by $\theta_{i_{1}}+2 \pi m_{i_{1}}=\cdots=\theta_{i_{M}}+2 \pi m_{i_{M}}$, $\left(m_{i_{1}}, \ldots, m_{i_{M}}\right) \in \mathbb{Z}^{M}$, are locally asymptotically stable under the flow of $(1)$.

\section{Discussion}

In this section we discuss some consequences of proposition 2. (Most of these observations also apply to proposition 1.)

Interpretation. If, in proposition 2 , the set $S_{1}$ contains a considerable fraction of the oscillators, $\alpha$ is sufficiently close to zero, and the other oscillators have natural frequencies that differ largely from those in $S_{1}$, such that $\delta$ is positive, then for sufficiently small frequency differences of the oscillators in $S_{1}$ a solution exhibiting partial entrainment with respect to $S_{1}$ is guaranteed to exist. If $|\alpha| \geq \frac{\pi}{6}$ a similar sufficient condition can still be derived by choosing $\tilde{s}$ - defined in the proof (see appendix) — in a different way.

Relation with proposition 1. To verify that proposition 2 is an extension of proposition 1 , set $\alpha=0,\left|S_{1}\right|=M, S_{2}=\emptyset$ and thus $\tilde{\gamma}_{2}=0$ and $T=1$ and assume that $M>\frac{N}{2}$ to obtain the condition (from proposition 2)

$$
\frac{\omega_{M}-\omega_{m}}{K} \leq \frac{4 \sqrt{3} \sqrt{N}(2 M-N)^{\frac{3}{2}}}{2(M+N)^{2}}
$$

Since

$$
\frac{4 \sqrt{3} \sqrt{N}(2 M-N)^{\frac{3}{2}}}{2(M+N)^{2}}=\frac{2 \sqrt{3} \sqrt{M}(3 N)^{\frac{3}{2}}}{2^{\frac{3}{2}}(M+N)^{2}} \sqrt{\frac{N}{M}}\left(\frac{4 M-2 N}{3 N}\right)^{\frac{3}{2}}
$$

with

$$
\frac{2 \sqrt{3} \sqrt{M}(3 N)^{\frac{3}{2}}}{2^{\frac{3}{2}}(M+N)^{2}} \geq \frac{2 \sqrt{3} \sqrt{\frac{N}{2}}(3 N)^{\frac{3}{2}}}{2^{\frac{3}{2}}(2 N)^{2}}=\frac{9}{8},
$$

it follows that proposition 2 entails proposition 1. 
Persistence under perturbations. Proposition 2 also implies that entrainment will persist under perturbations: for a perturbed initial condition $\tilde{\theta}(0)$, with $\tilde{\theta}(0) \in R_{a^{\prime}}$ and $\tilde{\theta}_{m}(0) \leq \tilde{\theta}_{i}(0) \leq \tilde{\theta}_{M}(0), \forall i \in S_{1}$, it follows from the proof that the entrainment with respect to $S_{1}$ will be maintained.

The limit $N \rightarrow \infty$. Notice also that proposition 2 remains non-trivial for $N \rightarrow \infty$ : if $S_{1}, S_{2}$ and $S_{3}$ contain oscillators with natural frequencies in prescribed intervals, then in general $\omega_{M}-\omega_{m}, \gamma_{1}, \tilde{\gamma}_{2}, \gamma_{3}, \delta$ and $T$ will approach non-zero constant values for $N \rightarrow \infty$.

In view of this remark, the persistence of the entrainment under perturbations may contribute to the understanding of the stability properties of the partially synchronized solutions for the system (1) with $N=\infty$. These stability properties are not yet fully understood.

Analytical identification of the entrainment behavior. The condition $\left|\omega_{i}-\omega_{j}\right|>2 K$ for some $i, j \in S_{1}$ is obviously sufficient to exclude partial entrainment with respect to $S_{1}$ since this implies that $\left|\theta_{i}(t)-\theta_{j}(t)\right|$ will grow unbounded. For some configurations this condition together with proposition 2 allows to determine a maximal entrained subset, i.e. a subset of $\{1, \ldots, N\}$ for which partial entrainment can occur and which is not included in another entrained subset. If the population can be partitioned in maximal entrained subsets, then the entire entrainment behavior can be determined on analytical grounds and all entrained subsets can be identified.

\section{Estimation of the transition values for $\alpha=0$}

The value of the coupling strength calculated from proposition 2, guaranteeing partial entrainment of a given subset, may be quite conservative. This is a consequence of the fact that only little information can be obtained about the interaction between oscillators from different entrained subsets. As a result, the discrepancy between the value calculated from proposition 2 for the entrainment of a subset $S_{\mathrm{e}}$ and the transition value for $K$ obtained from simulations decreases with increasing size of $S_{\mathrm{e}}$ (for the same population), as can be seen in figure 2 below.

In this section a better estimation for the actual transition value of the coupling strength is given for the case $\alpha=0$. 


\subsection{Estimation procedure}

Proposition 2 (and its proof) suggest(s) that oscillators which differ largely in natural frequency will have small mutual influence as to the entrainment behavior. Simulations confirm this and indicate that this is already true for much smaller frequency differences than suggested by the analytical results. We estimate the critical values for the coupling strength, defining the transitions between different forms of partial entrainment behavior, by neglecting the interactions between oscillators from different entrained subsets and using analytical results from [2]. Since the latter results are concerned with the case $\alpha=0$, we will restrict $\alpha$ to be zero throughout this section.

We estimate the entrainment behavior with respect to a subset $S_{\mathrm{e}}$ by disregarding all oscillators not belonging to $S_{\mathrm{e}}$, and we determine the coupling strength $K_{c}$ for which full entrainment of $S_{\mathrm{e}}$ would occur. This is done by numerically solving the following equations for $K$ and $r$, obtained from [2].

$$
r=\frac{1}{N} \sum_{j \in S_{\mathrm{e}}} \sqrt{1-\left(\frac{\omega_{j}^{\prime}}{K r}\right)^{2}}, \quad \sum_{j \in S_{\mathrm{e}}} \frac{1-2\left(\frac{\omega_{j}^{\prime}}{K r}\right)^{2}}{\sqrt{1-\left(\frac{\omega_{j}^{\prime}}{K r}\right)^{2}}}=0 .
$$

In these equations $\omega_{j}^{\prime} \triangleq \omega_{j}-\frac{1}{\left|S_{\mathrm{e}}\right|} \sum_{i \in S_{\mathrm{e}}} \omega_{i}$. The variable $r$ is - up to a factor $\frac{\left|S_{\mathrm{e}}\right|}{N}$ - equal to the order parameter corresponding to the subsystem (1) with $i \in S_{\mathrm{e}}$ and with the summation index $j$ also restricted to $S_{\mathrm{e}}$. For this subsystem the order parameter $r^{\prime}$ is defined as

$$
r^{\prime}(t) \triangleq\left|\frac{1}{\left|S_{\mathrm{e}}\right|} \sum_{j \in S_{\mathrm{e}}} e^{i \theta_{j}(t)}\right|, \quad \forall t \in \mathbb{R}
$$

and for $K$ sufficiently large, $r^{\prime}$ is time-invariant and $r \triangleq r^{\prime}(t) \frac{\left|S_{\mathrm{e}}\right|}{N}$ satisfies the first equation from (3). The second equation is satisfied at the transition to full entrainment and determines $K_{c}$. (See [2] for more details.)

This procedure is also supported by observations from the model with an infinite number of oscillators. For $\alpha=0$ and with the distribution $g$ of the natural frequencies even, the partially synchronized subset in the solution mentioned in the introduction is independent of the shape of the distribution $g$ outside the region corresponding to the frequencies of the oscillators in the partially synchronized subset. (See e.g. [16] for mathematical details.) 


\subsection{Comparison and discussion}

We compare the critical values for $K$ resulting from the above analytical estimation based on equalities (3) with the values for $K$ derived from propositions 1 and 2, and from simulations. We considered (1) for $\alpha=0$ and with $N=100$, and we randomly picked 100 natural frequencies from the distribution $g$ defined by

$$
g(\omega)= \begin{cases}C \frac{1-0.0001|\omega|}{0.0001+|\omega|}, & |\omega| \leq 10000 \\ 0, & |\omega|>10000\end{cases}
$$

where $C>0$ is such that $\int_{-\infty}^{+\infty} g(\omega) \mathrm{d} \omega=1$. (The expression for $g(\omega)$ is a slight modification of $1 / \omega$, to guarantee that $g$ can be normalized to 1 .) The frequencies were ordered by their absolute values.

For the calculation of the $K$-values according to propositions 1 and 2 and equation (3), we then considered the entrainment of the subsets $S_{\mathrm{e}}=\{1, \ldots, M\}$, with $M>1$. For each value of $M$, propositions 1 and 2 provide a minimal value of $K$ for which entrainment of $S_{\mathrm{e}}$ is guaranteed. Together with the estimation resulting from the equations (3) and the value given by the simulations, these are shown in figure 2 for varying $M$. In the simulations, the entrained subsets may differ from the sets $\{1, \ldots, M\}$, but in most cases they are equal. (In the case represented by figure 2 this holds for $95 \%$ of the $M$-values.)

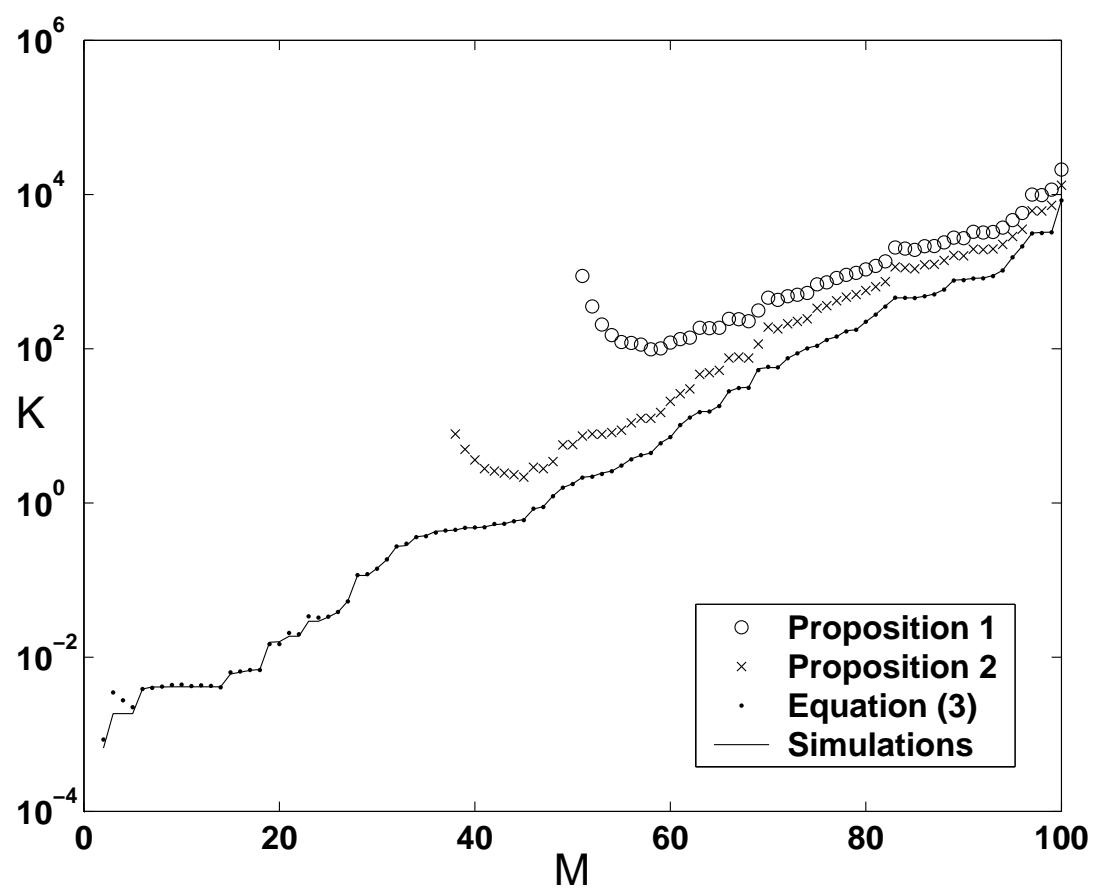

Fig. 2. Comparison of values for the coupling strength related to entrainment of the subset $\{1, \ldots, M\}$ for different procedures.

Since proposition 1 requires that $M>N / 2$, the corresponding curve only 
starts at $M=51$. Also proposition 2 does not generate results for any value of $M$, but the condition is less stringent and the corresponding curve starts for a value of $M<51$. (Although for several other distributions of the natural frequencies this curve also starts at $M=51$, the present form of $g$ shows that this does not always hold.) For the curve associated with proposition 2 the $K$-values are closer to those obtained from the simulations than for the curve associated with proposition 1 , but, considering the logarithmic scale, both are unsuitable as estimations for the transition values between different forms of partial entrainment.

For this choice of the natural frequencies, the estimation based on equations (3) corresponds quite well with the simulation results, justifying the assumption that oscillators outside an entrained subset have little influence on the entrainment of this subset.

\section{Entrainment break up with increasing $K$}

The general scenario does not always hold; one of the points we want to draw attention to is that entrained oscillators may break up with increasing $K$, a phenomenon reported before in $[8, \mathrm{p} .46]$ for the case $\alpha=0$. We consider a particular system with four oscillators, and also $\alpha=0$. Observe in figure 3 that there is a critical value for the coupling strength $( \pm 0.313)$ above which the entrainment of a subset breaks up. Further increase of $K$ reestablishes the entrainment.

We offer an intuitive explanation. Denote the oscillators by $1,2,3$ and 4 , according to the order of their natural frequencies $\omega_{i}$ (i.e. $\omega_{1}<\omega_{2}<\omega_{3}<\omega_{4}$ ).

As we have already mentioned in previous sections, the interaction between two oscillators appears to decrease with increasing difference in their natural frequencies. This implies that oscillator 3 will be subject to a stronger attraction towards oscillators 1 and 2 than oscillator 4 . With increasing coupling strength $K$ this attraction, and also the difference in attraction from oscillators 1 and 2 on oscillator 3 and on oscillator 4, will increase. For some value of $K$ oscillators 3 and 4 become entrained, but when $K$ is increased further, the increase in attraction difference becomes more important than the increased attraction between oscillators 3 and 4, making it possible for partial entrainment to disappear.

For $\alpha=0$ simulation results indicate that entrainment of two oscillators in a system of only three oscillators cannot disappear with increasing coupling strength. The probability of entrainment disappearing with increasing coupling strength seems to increase with $|\alpha|$, and for $|\alpha|$ sufficiently large it can 


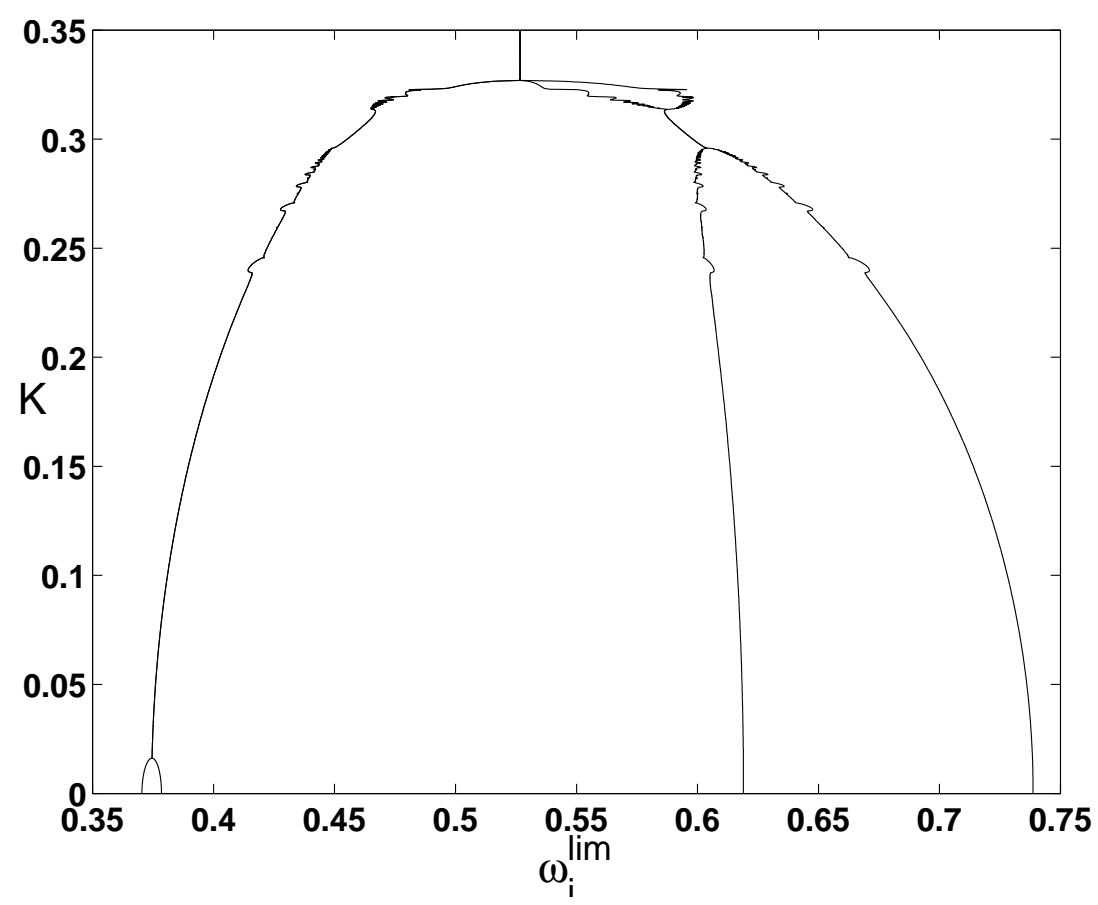

Fig. 3. Long term average frequencies for varying coupling strength for a system of four oscillators. When $K$ is increased from 0.3 to 0.35 there is a transition in which entrainment of two oscillators disappears. It reappears at the transition to full entrainment.

also be observed in a system of three oscillators.

\subsection{Persistence of full entrainment with increasing $K$}

The explanation offered in the previous paragraph implies that a given entrained subset can break up with increasing coupling strength only if other oscillators are present, and suggests that entrainment of the entire population cannot disappear with increasing coupling strength. For $\alpha=0$ this is confirmed as follows by analytical results that can be derived from [2]. In this paper, it was proven that the existence of a locally stable phase-locked solution (which corresponds to full entrainment) is equivalent with the existence of a solution $r \in(0,1]$ of (see also (3) in section 4 )

$$
\varphi_{1}(r, K) \triangleq r-\frac{1}{N} \sum_{i=1}^{N} \sqrt{1-\left(\frac{\omega_{i}^{\prime}}{K r}\right)^{2}}=0
$$


$\left(\right.$ with $\omega_{i}^{\prime} \triangleq \omega_{i}-\frac{1}{N} \sum_{j=1}^{N} \omega_{j} ; K r \geq \max _{i} \omega_{i}^{\prime}$ ), satisfying

$$
\varphi_{2}(r, K) \triangleq r-\frac{1}{N} \sum_{i=1}^{N} \frac{\left(\frac{\omega_{i}^{\prime}}{K r}\right)^{2}}{\sqrt{1-\left(\frac{\omega_{i}^{\prime}}{K r}\right)^{2}}}>0 .
$$

Assume that $r$ and $K$ satisfy both (4) and (5) and take $K^{\prime}>K$. It follows that $\varphi_{1}\left(r, K^{\prime}\right) \leq 0$, while clearly $\varphi_{1}\left(1, K^{\prime}\right) \geq 0$, implying the existence of an $r^{\prime} \in[r, 1]$ with $\varphi_{1}\left(r^{\prime}, K^{\prime}\right)=0$. From $r^{\prime} \geq r$ and $K^{\prime} r^{\prime}>K r$ and $\varphi_{1}\left(r^{\prime}, K^{\prime}\right)=0$ it then follows that

$$
\varphi_{2}\left(r^{\prime}, K^{\prime}\right) \geq \varphi_{2}(r, K)>0
$$

implying the existence of a solution of (1) for a coupling strength $K^{\prime}$ which exhibits entrainment of the entire population. This result implies that the solution of (1) corresponding to entrainment of the entire population will persist with increasing coupling strength (for the case $\alpha=0$ ).

\subsection{Josephson junctions}

The relation between the Kuramoto-Sakaguchi model and arrays of Josephson junctions [19] suggests that the phenomenon of destruction of entrainment with increasing $K$ may also be observed in Josephson junction arrays. Simulations confirm this. For a Josephson junction characterized by a phase difference $\phi$ the voltage and current across the junction are given by $\frac{\hbar}{2 e} \dot{\phi}$ and $I_{C} \sin \phi$ respectively, where $\hbar$ is Planck's constant divided by $2 \pi, e$ denotes the elementary electrical charge and the constant $I_{C}$ is the critical current of the junction. We consider the same circuit as in [19]: a (parallel) connection of a bias current $I_{B}, N$ different junctions in series, and a load with inductance $L$, resistance $R$ and capacitance $C$, with the charge on the capacitor denoted by $Q$. For junction $i$ the phase difference, resistance and critical current are denoted by $\phi_{i}, r_{i}$ and $I_{i}$ respectively; we assume its capacitance can be neglected. The system equations can then be written as

$$
\begin{aligned}
\frac{\hbar}{2 e r_{i}} \dot{\phi}_{i}+I_{i} \sin \phi_{i}+\dot{Q} & =I_{B}, \quad \forall i \in\{1, \ldots, N\}, \\
L \ddot{Q}+R \dot{Q}+\frac{Q}{C} & =\frac{\hbar}{2 e} \sum_{i=1}^{N} \dot{\phi}_{i} .
\end{aligned}
$$

In [19] it was shown that in the limit of weak coupling and weak disorder this system can be cast into the model (1). Setting $N=4, I_{B}=1.5 \mathrm{~mA}$, $R=50 \Omega, L=25 \mathrm{pH}, C=0.04 \mathrm{pF}, r_{i}=0.5 \Omega, \forall i \in\{1, \ldots, N\}$ and $I_{i}=$ $\left(0.5+\frac{I_{i}^{\prime}}{\beta_{I}}\right) \mathrm{mA}$, with $I^{\prime}=(-0.057,-0.021,0.0075,0.0165,0.021)$, (most values are taken from [19]), we calculate the long term average frequencies (i.e. the values $\left.\omega_{i}^{\lim } \triangleq \lim _{t \rightarrow \infty} \frac{\phi_{i}(t)-\phi_{i}(0)}{t}\right)$ for varying $\beta_{I}$, representing different levels 
of disorder. The result is shown in figure 4. In spite of the irregular shape of the graph (which persists when simulating with different time steps or over different time intervals, excluding simulation errors as the cause of the irregularity), different phenomena can be observed. Enumerating the junctions from 1 to 5 , such that $\omega_{1}^{\lim } \leq \omega_{2}^{\lim } \leq \omega_{3}^{\lim } \leq \omega_{4}^{\lim } \leq \omega_{5}^{\lim }$ (with strict inequalities e.g. for $\beta_{I}=9.6$, notice that for $\beta_{I}=9.2$ junctions 2 an 3 have already become entrained), the following transitions (with increasing $\beta_{I}$ ) are clearly visible:

- $\beta_{I} \approx 9.36$ junction 2 leaves junction 3 , and joins junction 1 at $\beta_{I} \approx 9.71$;

- $\beta_{I} \in(10.03,10.16)$ : entrainment of junctions 1 and 2 temporarily disappears;

- $\beta_{I} \in(9.31,10)$ : junctions 4 and 5 are entrained but for several subintervals the entrainment disappears;

- $\beta_{I} \approx 10.17$ : junction 4 (having left junction 5 at $\beta_{I} \approx 10$ ) becomes entrained with junction 3 .

\section{Conclusion}

We have investigated the partial entrainment behavior of the KuramotoSakaguchi model. We derived a sufficient condition for partial entrainment of a given subset of oscillators; the result implies persistence of the entrainment behavior under perturbations and remains non-trivial in the limit $N \rightarrow \infty$.

For the investigated distribution of natural frequencies, the critical value of the coupling strength defining the onset of partial entrainment of a given subset can be estimated analytically by neglecting oscillators which do not belong to this subset.

Simulations indicate that entrainment can disappear with increasing coupling strength in the Kuramoto-Sakaguchi model, and that a similar phenomenon can be observed in arrays of Josephson junctions, where it is also possible that a junction leaving one entrained subset joins another entrained subset.

\section{Acknowledgments}

This paper presents research results of the Belgian Programme on Interuniversity Attraction Poles, initiated by the Belgian Federal Science Policy Office. The scientific responsibility rests with its authors.

Filip De Smet is a Research Assistant of the Research Foundation - Flanders (FWO - Vlaanderen). 


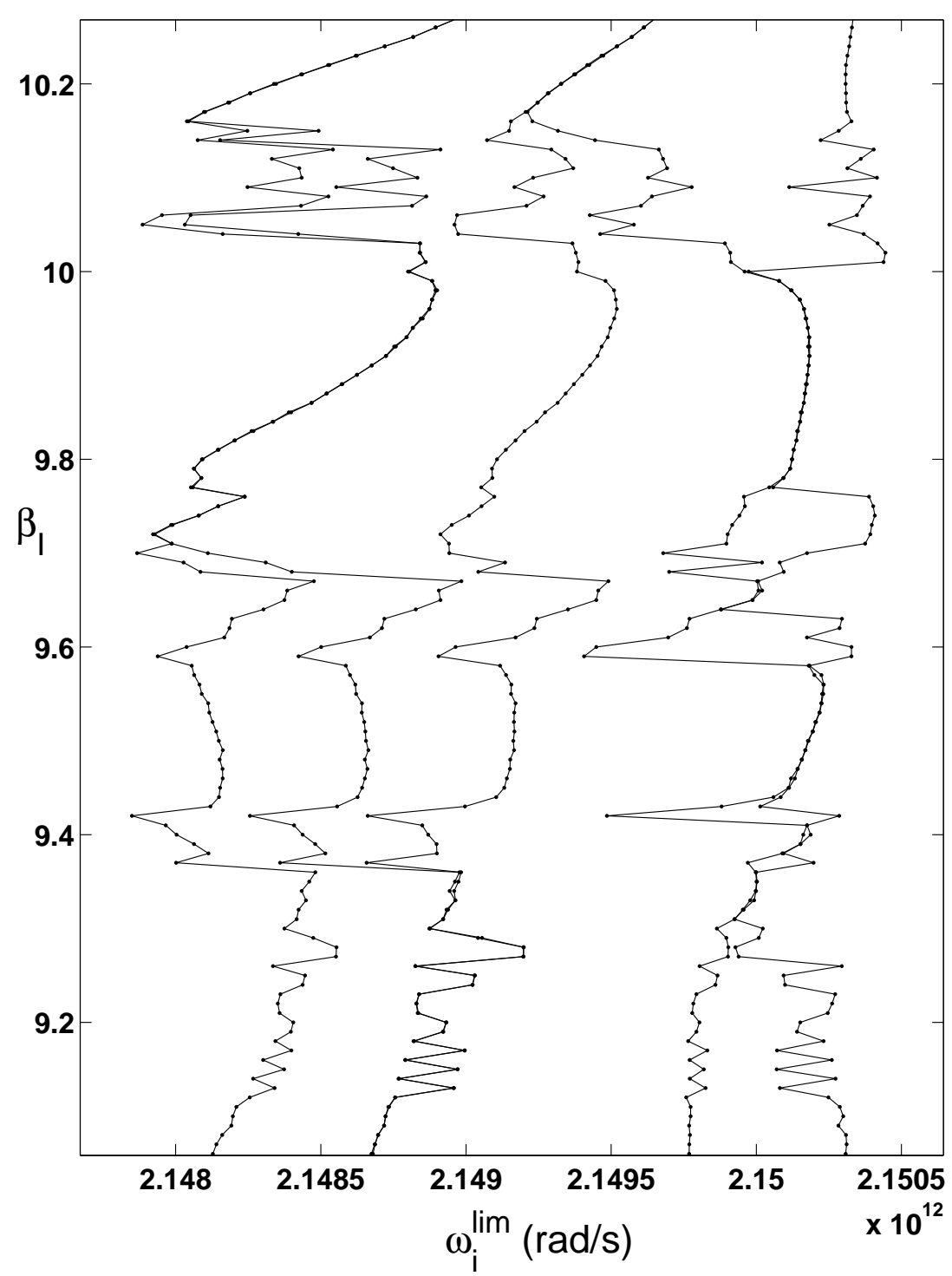

Fig. 4. Long term average frequencies for varying levels of disorder for an array of 5 Josephson junctions. When $\beta_{I}$ is increased there are different transitions in which entrainment of two junctions disappears.

\section{A Proof of proposition 2}

The main idea of the proof is similar to the idea behind the proof of proposition 1. Instead of one trapping region, we will consider two regions and show that a solution starting in the smaller region cannot leave the larger region (which encompasses the smaller region). We first formulate and prove a lemma that allows us to provide a better bound for the term $\cos \left(\theta_{k}\left(t_{0}\right)-\frac{\theta_{i}\left(t_{0}\right)+\theta_{j}\left(t_{0}\right)}{2}\right)$ in (2) with $k \notin S_{\mathrm{e}}$.

Lemma 1. Assume the continuously differentiable function $\theta_{0}: \mathbb{R} \rightarrow \mathbb{R}: t \mapsto$ 
$\theta_{0}(t)$ satisfies $0<\Omega_{1} \leq\left|\dot{\theta}_{0}(t)\right| \leq \Omega_{2}, \forall t \in \mathbb{R}^{+}$. Then

$$
\int_{0}^{t} \cos \theta_{0}\left(t^{\prime}\right) \mathrm{d} t^{\prime} \leq t \cos \Theta_{0}+\frac{2}{\Omega_{1}}\left(\sin \Theta_{0}-\Theta_{0} \cos \Theta_{0}\right)
$$

for all $\Theta_{0} \in\left(0, \frac{\pi}{2}\right)$ satisfying

$$
2\left(\frac{1}{\Omega_{1}}-\frac{1}{\Omega_{2}}\right)\left(\sin \Theta_{0}-\Theta_{0} \cos \Theta_{0}\right)-\frac{2 \pi}{\Omega_{2}} \cos \Theta_{0} \leq 0 .
$$

Proof. The result is invariant under the substitution $\theta_{0} \leftrightarrow-\theta_{0}$, and therefore we will only consider the case for which $\dot{\theta}(t)>0, \forall t \in \mathbb{R}^{+}$. Since $\theta_{0}$ is strictly increasing we can perform the substitution $t^{\prime}=\theta_{0}^{-1}\left(\theta_{0}^{\prime}\right)$ in the following integral.

$$
\int_{0}^{t}\left(\cos \theta_{0}\left(t^{\prime}\right)-\cos \Theta_{0}\right) \mathrm{d} t^{\prime}=\int_{\theta_{0}(0)}^{\theta_{0}(t)} \frac{\left(\cos \theta_{0}^{\prime}-\cos \Theta_{0}\right) \mathrm{d} \theta_{0}^{\prime}}{\dot{\theta}_{0}\left(\theta_{0}^{-1}\left(\theta_{0}^{\prime}\right)\right)}
$$

Setting $I_{+}(t) \triangleq\left\{\theta_{0}^{\prime} \in\left[0, \theta_{0}(t)\right]: \cos \left(\theta_{0}^{\prime}\right) \geq \cos \Theta_{0}\right\}$ and $I_{-}(t) \triangleq\left\{\theta_{0}^{\prime} \in\right.$ $\left.\left[0, \theta_{0}(t)\right]: \cos \left(\theta_{0}^{\prime}\right) \leq \cos \Theta_{0}\right\}$, we obtain the inequality

$$
\begin{aligned}
\int_{0}^{t} \cos \theta_{0}\left(t^{\prime}\right) \mathrm{d} t^{\prime}-t \cos \Theta_{0} \leq \int_{I_{+}(t)} \frac{\left(\cos \theta_{0}^{\prime}-\cos \Theta_{0}\right) \mathrm{d} \theta_{0}^{\prime}}{\Omega_{1}} \\
+\int_{I_{-}(t)} \frac{\left(\cos \theta_{0}^{\prime}-\cos \Theta_{0}\right) \mathrm{d} \theta_{0}^{\prime}}{\Omega_{2}}
\end{aligned}
$$

Denoting by $n(t)$ the number of connected components of $I_{+}(t)$ if $I_{+}(t) \neq \emptyset$ and setting $n(t) \triangleq 1$ for $I_{+}(t)=\emptyset$ we obtain

$$
\begin{aligned}
\int_{0}^{t} \cos \theta_{0}\left(t^{\prime}\right) \mathrm{d} t^{\prime}-t \cos \Theta_{0} \leq & \frac{n(t)}{\Omega_{1}} \int_{-\Theta_{0}}^{\Theta_{0}}\left(\cos \theta_{0}^{\prime}-\cos \Theta_{0}\right) \mathrm{d} \theta_{0}^{\prime} \\
& \quad+\frac{n(t)-1}{\Omega_{2}} \int_{\Theta_{0}}^{2 \pi-\Theta_{0}}\left(\cos \theta_{0}^{\prime}-\cos \Theta_{0}\right) \mathrm{d} \theta_{0}^{\prime} \\
= & \frac{2 n(t)}{\Omega_{1}}\left(\sin \Theta_{0}-\Theta_{0} \cos \Theta_{0}\right) \\
& \quad-\frac{2(n(t)-1)}{\Omega_{2}}\left(\sin \Theta_{0}+\left(\pi-\Theta_{0}\right) \cos \Theta_{0}\right) \\
= & \frac{2}{\Omega_{1}}\left(\sin \Theta_{0}-\Theta_{0} \cos \Theta_{0}\right)+(n(t)-1) \\
& \times\left(2\left(\frac{1}{\Omega_{1}}-\frac{1}{\Omega_{2}}\right)\left(\sin \Theta_{0}-\Theta_{0} \cos \Theta_{0}\right)-\frac{2 \pi}{\Omega_{2}} \cos \Theta_{0}\right),
\end{aligned}
$$

proving the lemma.

We will now prove proposition 2 . 
For an $a \in(0, \pi-2|\alpha|)$, yet to be determined, consider the region

$$
R_{a} \triangleq\left\{\theta \in \mathbb{R}^{N}:\left|\theta_{i}-\theta_{j}\right| \leq a, \forall i, j \in S_{1}\right\}
$$

We will determine conditions such that a well-chosen initial condition leads to a solution $\theta$ of (1) which remains in $R_{a}$. The initial value $\theta(0)$ belongs to $R_{a^{\prime}}$, with $a^{\prime} \in(0, a)$ also yet to be determined, and is such that $\theta_{m}(0) \leq \theta_{i}(0) \leq$ $\theta_{M}(0), \forall i \in S_{1}$. This implies that $\theta_{m}(t) \leq \theta_{i}(t) \leq \theta_{M}(t), \forall t \in \mathbb{R}^{+}$, since if for some $t^{*} \geq 0, \theta_{i}\left(t^{*}\right)=\theta_{m}\left(t^{*}\right)$ (resp. $\left.\theta_{i}\left(t^{*}\right)=\theta_{M}\left(t^{*}\right)\right)$, for some $i \in S_{1}$, then $\dot{\theta}_{i}\left(t^{*}\right)-\dot{\theta}_{m}\left(t^{*}\right)=\omega_{i}-\omega_{m} \geq 0$ (resp. $\left.\dot{\theta}_{i}\left(t^{*}\right)-\dot{\theta}_{M}\left(t^{*}\right) \leq 0\right)$. Consequently we only need to derive conditions guaranteeing that $\theta_{M}(t)-\theta_{m}(t)$ will remain smaller than or equal to $a$. With $\varphi \triangleq \frac{\theta_{M}-\theta_{m}}{2}$ and $\omega \triangleq \frac{\omega_{M}-\omega_{m}}{2}$ it follows that

$$
\dot{\varphi}(t)=\omega-\frac{K}{N} \sin \varphi(t) \sum_{j=1}^{N} \cos \left(\theta_{j}(t)-\frac{\theta_{m}(t)+\theta_{M}(t)}{2}-\alpha\right) .
$$

We will investigate the behavior of $\varphi$ for $t$-values for which $\varphi(t) \in\left[\frac{a^{\prime}}{2}, \frac{a}{2}\right]$. In this situation the terms in the summation for which $j \in S_{1}$ are bounded by

$$
\cos \left(\theta_{j}(t)-\frac{\theta_{m}(t)+\theta_{M}(t)}{2}-\alpha\right) \geq \cos \left(\frac{a}{2}+|\alpha|\right),
$$

since $\theta_{m}(t) \leq \theta_{j}(t) \leq \theta_{M}(t)$ and thus $\left|\theta_{j}(t)-\frac{\theta_{m}(t)+\theta_{M}(t)}{2}\right| \leq \frac{a}{2}$. If $j \in S_{3}$, we apply that $\cos \left(\theta_{j}(t)-\frac{\theta_{m}(t)+\theta_{M}(t)}{2}-\alpha\right) \geq-1$, and thus

$$
\begin{aligned}
\dot{\varphi}(t) \leq \omega-\frac{K}{N} \sin \varphi(t)\left(\left|S_{1}\right| \cos \left(\frac{a}{2}+|\alpha|\right)-\left|S_{3}\right|\right. & \\
& \left.+\sum_{j \in S_{2}} \cos \left(\theta_{j}(t)-\frac{\theta_{m}(t)+\theta_{M}(t)}{2}-\alpha\right)\right) .
\end{aligned}
$$

With $\varphi(t) \in\left[\frac{a^{\prime}}{2}, \frac{a}{2}\right]$, and $\gamma_{i}=\frac{\left|S_{i}\right|}{N}(i \in\{1,3\})$, we obtain

$$
\begin{aligned}
\frac{\dot{\varphi}(t)}{K \sin \varphi(t)} \leq \frac{\omega}{K \sin \frac{a^{\prime}}{2}}-\gamma_{1} \cos \left(\frac{a}{2}+|\alpha|\right) & +\gamma_{3} \\
& -\frac{1}{N} \sum_{j \in S_{2}} \cos \left(\theta_{j}(t)-\frac{\theta_{m}(t)+\theta_{M}(t)}{2}-\alpha\right),
\end{aligned}
$$

or

$$
\begin{aligned}
\frac{1}{K} \ln \left(\frac{\tan \frac{\varphi(t)}{2}}{\tan \frac{\varphi\left(t_{0}\right)}{2}}\right) \leq & \left(\frac{\omega}{K \sin \frac{a^{\prime}}{2}}-\gamma_{1} \cos \left(\frac{a}{2}+|\alpha|\right)+\gamma_{3}\right)\left(t-t_{0}\right) \\
& -\frac{1}{N} \sum_{j \in S_{2}} \int_{t_{0}}^{t} \cos \left(\theta_{j}\left(t^{\prime}\right)-\frac{\theta_{m}\left(t^{\prime}\right)+\theta_{M}\left(t^{\prime}\right)}{2}-\alpha\right) \mathrm{d} t^{\prime}
\end{aligned}
$$


for all $t_{0}, t \geq 0$, such that $\varphi\left(t^{\prime}\right) \in\left[\frac{a^{\prime}}{2}, \frac{a}{2}\right], \forall t^{\prime} \in\left[t_{0}, t\right]$. We want to establish conditions for which the right hand side is upper bounded by $\frac{1}{K} \ln \left(\frac{\tan \frac{a}{4}}{\tan \frac{a^{\prime}}{4}}\right)$, guaranteeing that for increasing $t, \varphi(t)$ can only leave the interval $\left[\frac{a^{\prime}}{2}, \frac{a}{2}\right]$ by becoming smaller than $\frac{a^{\prime}}{2}$.

Consider a $j \in S_{2}$. Then

$$
\begin{array}{r}
0<\left|\omega_{j}-\frac{\omega_{m}+\omega_{M}}{2}\right|-2 K \leq\left|\dot{\theta}_{j}(t)-\frac{\dot{\theta}_{m}(t)+\dot{\theta}_{M}(t)}{2}\right| \\
\leq\left|\omega_{j}-\frac{\omega_{m}+\omega_{M}}{2}\right|+2 K
\end{array}
$$

With the same notation as in lemma 1 , define $\mathcal{F}_{\Omega_{1}, \Omega_{2}}:[0,1] \rightarrow \mathbb{R}$ by

$$
\begin{aligned}
\mathcal{F}_{\Omega_{1}, \Omega_{2}}\left(\cos \Theta_{0}\right) & \triangleq 2\left(\frac{1}{\Omega_{1}}-\frac{1}{\Omega_{2}}\right)\left(\sin \Theta_{0}-\Theta_{0} \cos \Theta_{0}\right)-\frac{2 \pi}{\Omega_{2}} \cos \Theta_{0} \\
& \leq 0
\end{aligned}
$$

$\forall \Theta_{0} \in\left[0, \frac{\pi}{2}\right]$. Notice that $\mathcal{F}_{\Omega_{1}, \Omega_{2}}(0) \geq 0, \mathcal{F}_{\Omega_{1}, \Omega_{2}}(1)<0$, and one can also verify that $\mathcal{F}_{\Omega_{1}, \Omega_{2}}^{\prime \prime}\left(\Theta_{0}\right) \geq 0, \forall \Theta_{0} \in\left[0, \frac{\pi}{2}\right]$. It follows that the function $\mathcal{F}_{\Omega_{1}, \Omega_{2}}$ is convex and that the value $\cos \tilde{\Theta}_{0}$ for which the linear interpolation between $\left(0, \mathcal{F}_{\Omega_{1}, \Omega_{2}}(0)\right)$ and $\left(1, \mathcal{F}_{\Omega_{1}, \Omega_{2}}(1)\right)$ will become zero will satisfy $\mathcal{F}_{\Omega_{1}, \Omega_{2}}\left(\cos \tilde{\Theta}_{0}\right) \leq$ 0 . It can be calculated as

$$
\cos \tilde{\Theta}_{0}=\frac{\Omega_{2}-\Omega_{1}}{\Omega_{2}-\Omega_{1}+\pi \Omega_{1}} .
$$

In order to apply lemma 1 to $\theta_{j}$, with $j \in S_{2}, \Omega_{1}$ and $\Omega_{2}$ have to replaced by $\left|\omega_{j}-\frac{\omega_{m}+\omega_{M}}{2}\right|-2 K$ and $\left|\omega_{j}-\frac{\omega_{m}+\omega_{M}}{2}\right|+2 K$ respectively. By defining $\Theta_{j}$ as

$$
\tilde{\Theta}_{j} \triangleq \arccos \left(\frac{4 K}{4 K+\pi\left(\left|\omega_{j}-\frac{\omega_{m}+\omega_{M}}{2}\right|-2 K\right)}\right)
$$

$\forall j \in S_{2}$, and applying lemma 1 we obtain the following inequality:

$$
\begin{array}{r}
\frac{1}{K} \ln \left(\frac{\tan \frac{\varphi(t)}{2}}{\tan \frac{\varphi\left(t_{0}\right)}{2}}\right) \leq\left(\frac{\omega}{K \sin \frac{a^{\prime}}{2}}-\gamma_{1} \cos \left(\frac{a}{2}+|\alpha|\right)+\gamma_{3}+\frac{1}{N} \sum_{j \in S_{2}} \cos \tilde{\Theta}_{j}\right)\left(t-t_{0}\right) \\
\left.+\frac{1}{N} \sum_{j \in S_{2}} \frac{2\left(\sin \tilde{\Theta}_{j}-\tilde{\Theta}_{j} \cos \tilde{\Theta}_{j}\right)}{\left|\omega_{j}-\frac{\omega_{m}+\omega_{M}}{2}\right|-2 K}, \quad \text { (A. } 1\right)
\end{array}
$$


$\forall t_{0}, t \in \mathbb{R}^{+}: \varphi\left(\left[t_{0}, t\right]\right) \subset\left[\frac{a^{\prime}}{2}, \frac{a}{2}\right]$. By requiring that

$$
\frac{\omega}{K \sin \frac{a^{\prime}}{2}}-\gamma_{1} \cos \left(\frac{a}{2}+|\alpha|\right)+\gamma_{3}+\tilde{\gamma}_{2} \leq 0,
$$

and

$$
\frac{1}{K} \ln T \leq \frac{1}{K} \ln \left(\frac{\tan \frac{a}{4}}{\tan \frac{a^{\prime}}{4}}\right)
$$

it is guaranteed that $\varphi(t) \leq \frac{a}{2}, \forall t \in \mathbb{R}^{+}$, (keeping in mind the choice of the initial condition) and that there is partial entrainment with respect to $S_{1}$. Set $a^{\prime} \triangleq 4 \arctan \left(\frac{\tan \frac{a}{4}}{T}\right)$, automatically fulfilling (A.2b). Inequality (A.2a) then becomes

$$
\frac{\omega\left(T^{2}+\tan ^{2} \frac{a}{4}\right)}{2 K T \tan \frac{a}{4}}-\gamma_{1} \cos \left(\frac{a}{2}+|\alpha|\right)+\tilde{\gamma}_{2}+\gamma_{3} \leq 0,
$$

or, setting $s \triangleq \tan \frac{a}{4} \in\left(0, \tan \left(\frac{\pi}{4}-\frac{|\alpha|}{2}\right)\right)$,

$\frac{\omega\left(T^{2}+s^{2}\right)\left(1+s^{2}\right)}{2 K T}-\gamma_{1} s\left(\left(1-s^{2}\right) \cos \alpha-2 s|\sin \alpha|\right)+s\left(1+s^{2}\right)\left(\tilde{\gamma}_{2}+\gamma_{3}\right) \leq 0$.

Each value of $s \in\left(0, \tan \left(\frac{\pi}{4}-\frac{|\alpha|}{2}\right)\right)$ leads to a sufficient condition for the existence of a solution which is partially entrained with respect to $S_{1}$. To obtain the least stringent condition, one could take the derivative of the left hand side with respect to $s$ and then substitute the appropriate root of the resulting third order polynomial in the inequality. To avoid obtaining too complicated expressions we take the derivative of the left hand side and we calculate the (non-negative) root $\tilde{s}$ under the assumption that $\omega$ is equal to zero and in the absence of the term in $|\sin \alpha|$ :

$$
\tilde{s}=\sqrt{\frac{\gamma_{1} \cos \alpha-\tilde{\gamma}_{2}-\gamma_{3}}{3\left(\gamma_{1} \cos \alpha+\tilde{\gamma}_{2}+\gamma_{3}\right)}} .
$$

Substitution of $\tilde{s}$ in the previous inequality leads to proposition 2 .

\section{B Proof of proposition 3}

We can repeat the derivation of the previous section with $\omega=0$, leading to the inequality (A.1) which now holds for all $t_{0}, t \in \mathbb{R}^{+}$for which $\varphi\left(t_{0}, t\right) \subset\left(0, \frac{a}{2}\right]$. By demanding $K$ to be sufficiently small the summation $\sum_{j \in S_{2}} \cos \tilde{\Theta}_{j}$ in (A.1) can be made as small as needed and again setting $a^{\prime} \triangleq 4 \arctan \left(\frac{\tan \frac{a}{4}}{T}\right),(\mathrm{A} .2 \mathrm{~b})$ can be satisfied. Since $\gamma_{1} \cos \alpha>\gamma_{3}$, (A.2a) holds with strict inequality if $a$ and $K$ are chosen sufficiently small. From (A.1) we then obtain that $\lim _{t \rightarrow \infty} \varphi(t)=$ 0 , leading to proposition 3 . 


\section{References}

[1] J. A. Acebrón, L. L. Bonilla, C. J. P. Vicente, F. Ritort, and R. Spigler. The Kuramoto model: a simple paradigm for synchronization phenomena. Reviews of Modern Physics, 77(1):137-185, January 2005.

[2] D. Aeyels and J. A. Rogge. Existence of partial entrainment and stability of phase locking behavior of coupled oscillators. Progress of Theoretical Physics, 112(6):921-942, December 2004.

[3] H. Daido. Lower critical dimension for populations of oscillators with randomly distributed frequencies: a renormalization-group analysis. Physical Review Letters, 61(2):231-234, July 1988.

[4] H. Hong, M. Y. Choi, and B. J. Kim. Synchronization on small-world networks. Physical Review E, 65(2):026139, January 2002.

[5] A. Jadbabaie, N. Motee, and M. Barahona. On the stability of the Kuramoto model of coupled nonlinear oscillators. In Proceedings of the American Control Conference (ACC 2004), volume 5, pages 4296-4301, July 2004.

[6] Y. Kuramoto. Cooperative dynamics of oscillator community. Supplement of the Progress of Theoretical Physics, 79:223-240, 1984.

[7] Y. Maistrenko, O. Popovych, O. Burylko, and P. A. Tass. Mechanism of desynchronization in the finite-dimensional Kuramoto model. Physical Review Letters, 93(8):084102, August 2004.

[8] S. C. Manrubia, A. S. Mikhailov, and D. H. Zanette. Emergence of Dynamical Order: Synchronization Phenomena in Complex Systems, volume 2 of World Scientific Lecture Notes in Complex Systems. World Scientific, 2004.

[9] M. Maródi, F. d'Ovidio, and T. Vicsek. Synchronization of oscillators with long range interaction: phase transition and anomalous finite size effects. Physical Review E, 66(1):011109, 2002.

[10] L. G. Morelli, H. A. Cerdeira, and D. H. Zanette. Frequency clustering of coupled phase oscillators on small-world networks. European Physical Journal B, 43(2):243-250, January 2005.

[11] Y. Moreno and A. F. Pacheco. Synchronization of Kuramoto oscillators in scale-free networks. Europhysics Letters, 68(4):603-609, November 2004.

[12] O. V. Popovych, Y. L. Maistrenko, and P. A. Tass. Phase chaos in coupled oscillators. Physical Review E, 71(6):065201(R), June 2005.

[13] J. L. Rogers and L. T. Wille. Phase transitions in nonlinear oscillator chains. Physical Review E, 54(3):R2193-R2196, September 1996.

[14] J. A. Rogge and D. Aeyels. Stability of phase locking in a ring of unidirectionally coupled oscillators. Journal of Physics A: Mathematical and General, 37(46):11135-11148, November 2004.

[15] H. Sakaguchi and Y. Kuramoto. A soluble active rotator model showing phase transitions via mutual entrainment. Progress of Theoretical Physics, 76(3):576-581, September 1986.

[16] S. H. Strogatz. From Kuramoto to Crawford: exploring the onset of 
synchronization in populations of coupled oscillators. Physica D, 143:120, 2000.

[17] S. H. Strogatz. SYNC: The Emerging Science of Spontaneous Order. Hyperion Press, 2003.

[18] S. Watanabe and S. H. Strogatz. Constants of motion for superconducting Josephson arrays. Physica D, 74(3-4):197-253, July 1994.

[19] K. Wiesenfeld, P. Colet, and S. H. Strogatz. Frequency locking in Josephson arrays: Connection with the Kuramoto model. Physical Review E, 57(2):1563-1569, February 1998. 